Research Article

\title{
Rolling Scheduling Method considering Shiftable Demand Respond Resources
}

\author{
Zhengchong Lin, ${ }^{1}$ Feng Gao, ${ }^{1}$ Junwen Song, ${ }^{1}$ Changsheng Lin, ${ }^{1}$ Boyang Xu, ${ }^{1}$ Qiliang Liu, ${ }^{2}$ \\ Weizhuo Qiao, ${ }^{2}$ and Yuqing Bao $\mathbb{D D}^{2}$ \\ ${ }^{1}$ Shenzhen Power Supply Bureau Co. Ltd., Shenzhen 518000, China \\ ${ }^{2}$ School of Electrical Engineering, Southeast University, Nanjing 210096, China \\ Correspondence should be addressed to Yuqing Bao; 503000747@qq.com
}

Received 15 January 2021; Revised 8 February 2021; Accepted 12 February 2021; Published 23 February 2021

Academic Editor: François Vallée

Copyright (c) 2021 Zhengchong Lin et al. This is an open access article distributed under the Creative Commons Attribution License, which permits unrestricted use, distribution, and reproduction in any medium, provided the original work is properly cited.

In order to deal with the fluctuation of the renewable energy, this paper proposes rolling scheduling strategy taking into account the capacity of load-side resources. By considering the energy characteristics of shiftable loads, an improved rolling scheduling model is proposed by adopting a full-cycle scheduling and adding periodic power constraints. By this way, the accuracy of the scheduling can be improved. The testing examples verified that the proposed rolling scheduling method can reflect the long-time benefit and therefore result in better performance.

\section{Introduction}

The increasing penetration of renewable energy is conducive to sustainable economic development and the achievement of the "carbon neutrality" target [1]. However, the uncertainty, high fluctuations, and antipeak shaving characteristics of new energy generation seriously affect the safe and stable operation of the grid [2]. Using conventional generating units to participate in grid regulation has become more difficult, and the potential for demand-side resources to contribute to grid regulation needs to be explored [3].

To accommodate the uncertainty of new energy generation such as wind power, rolling scheduling of the grid is required according to the load forecasting accuracy on different timescales. Qiu et al. in [4] proposed a two-stage scheduling model combining improved day-ahead distributional robust optimization with intraday multi-timescale rolling optimization, which reduces the impact of new energy generation uncertainty on dispatch and improves the economic efficiency of scheduling. Yang et al. in [5] developed a multi-timescale model considering demand respond (DR) classification for hierarchical scheduling of multiple energy sources based on differences in scheduling times. Yuan et al. in [6] explored the potential for coordinated optimization of multiple units as well as price-based and incentive-based DRs at different timescales based on source-load uncertainty and constructs a rolling scheduling of "day-ahead intraday real-time" optimization model.

The above references show that DR program is an important option for maintaining a balance between supply and demand on the grid. DR load is divided into curtailable load and shiftable load. In contrast to other flexible load resources, shiftable load has the flexibility to change its energy-using periods but usually needs to meet the same total energy over the entire operating cycle as before the shift. Therefore, the power constraint of its participation in the DR needs to be considered. A great deal of research has been carried out around shiftable loads. A multiobjective optimization model for isolated microgrids accounting for shiftable loads is developed in [7], where shiftable loads are incorporated into the system optimization to smooth the output of new energy generators and improve the intermittent energy utilization of the microgrid. An integrated resource planning model that considers the impact of 
transferable loads in microgrids is proposed in [8] to reduce the cost of microgrid planning. To address the difficulties in solving purely mathematical exact modelling of shiftable loads, practical approximation methods and heuristic approaches are proposed to achieve the management of large amounts of shiftable loads in [9].

The above references take advantage of the characteristics of DR to effectively accommodate wind power and incorporate it in rolling scheduling. However, for shiftable DR resources, the scheduling characteristics are restricted by the energy storage constraint, while the abovementioned references take a fixed scheduling period for the intraday scheduling, which is not applicable to energy storage constraint.

To fill this gap, this paper proposes an improved method for the participation of shiftable load in rolling scheduling, which is optimally solved using variable period scheduling, considering the total cost of scheduling for the remaining time period, rather than being limited to considering the cost of scheduling for a fixed scheduling interval. The improved intraday scheduling of shiftable loads takes into account both the overall benefits and better matches the results of day-ahead scheduling.

The contributions of this paper can be summarized as follows:

(1) Rolling scheduling considering shiftable load. A dayahead intraday rolling scheduling strategy is proposed considering the energy constraint of the shiftable load.

(2) Variable intraday scheduling period. The intraday scheduling uses the remaining time of the day as the scheduling period, instead of the next 2 hours. By this way, long-term benefits can be reflected.

The remaining sections of the paper are organized as follows. Section 2 presents the improved rolling scheduling model, Section 3 performs the model simulation and solution, and Section 4 concludes the paper.

\section{Rolling Scheduling Method}

2.1. Framework of Rolling Scheduling. In a real electricity market such as Guangdong electricity market in China, the market can be divided into two parts: day-ahead market and intraday (or real-time) market. As a supplementary to dayhead market, the intraday market utilizes recent (within 1-4 hours) load forecasting data to obtain more precise scheduling results. The market clearing period of intraday market is usually set to 15 minutes. The day-ahead scheduling and intraday scheduling are implemented by dayahead market and intraday market, respectively.

The framework of the rolling scheduling is shown in Figure 1. The day-ahead scheduling executes every 24 hours, determining the allocation of resources of the whole day. The intraday scheduling executes every 2 hours, determining the allocation of resources of the next 2 hours. By the rolling scheduling, the intraday scheduling can utilize more accurate intraday load forecasting results and make the scheduling more accurate.

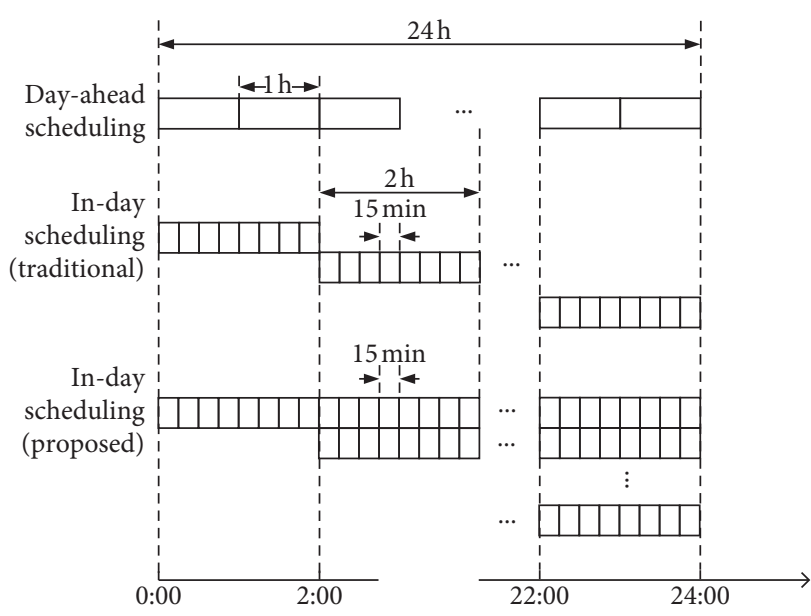

FIgURE 1: The framework of the rolling scheduling.

However, traditional intraday scheduling only utilizes the forecasted load of its own scheduling period (the next 2 hours), and the rest periods of the day are not considered. This way of scheduling works well for resources like generators but is not suitable for shiftable loads. It is because shiftable loads are constrained by the energy, which is time correlated. The short-term benefit may be contrary to the long-time benefit. To overcome this problem, a whole-horizon intraday scheduling method is proposed. By the proposed method, not only the next 2hour scheduling is considered, but also the period to the end of the day is taken into concern. By this way, the intraday scheduling can give consideration to the longtime benefit and better cooperate with day-ahead scheduling. The difference between the proposed rolling scheduling method and the traditional intraday scheduling method is shown in Figure 1.

2.2. Day-Ahead Scheduling. The day-ahead scheduling adopts scenario-based stochastic programming. Scenariobased stochastic programming is a useful method to cope with the scheduling considering uncertainties. Unlike deterministic scheduling, the scenario-based scheduling generates a set of load and wind power scenarios instead of a single deterministic forecasted load. Each scenario is subjected to all the constraints of the scheduling model, and the overall objective is the weighted sum of the objective under all the scenarios. By this way, the scheduling can give consideration to different scenarios and making the scheduling results more robust. More details of the scenarios-based can be found in [10].

The objective of day-ahead scheduling is minimizing the operation cost. The objective function can be formulized as follows:

$$
\min \left(f_{\mathrm{DA}}\right)=f_{\mathrm{G}}+f_{\mathrm{SL}},
$$

where $f_{\mathrm{G}}$ is the cost for the generators and $f_{\mathrm{SL}}$ is the cost for the shiftable load.

The generation cost $f_{\mathrm{G}}$ takes the following expression [11]: 


$$
f_{\mathrm{G}}=\sum_{i=1}^{N_{\mathrm{G}}} \sum_{t=1}^{N_{T}}\left(\sum_{s=1}^{N_{S}} \pi_{s} \cdot\left(a_{i}+b_{i} P_{i, s, t}+c_{i} P_{i, s, t}^{2}\right)+\mathrm{SU}_{i, t}+\mathrm{SD}_{i, t}\right),
$$

where $\pi_{s}$ is the possibility of the sth scenario, $P_{i, s, t}$ is the output power of the $i$ th generator in the sth scenario at time $t, a_{i}, b_{i}$, and $c_{i}$ are the coefficients of the fuel cost of the $i$ th generator, $\mathrm{SU}_{i, t}$ and $\mathrm{SD}_{i, t}$ are the start-up and shutdown cost of the $i$ th generator at time $t$, and $N_{G}, N_{T}$, and $N_{S}$ are the number of generators, the number of time periods, and the number of scenarios in the scheduling, respectively.

The cost for the shiftable load can be written as follows:

$$
f_{\mathrm{SL}}=\sum_{l=1}^{N_{L}} \sum_{t=1}^{N_{T}} \sum_{s=1}^{N_{S}} \pi_{s}\left(p_{l, t}^{+} \cdot L_{l, s, t}^{+}+p_{l, t}^{-} \cdot L_{l, s, t}^{-}\right)
$$

where $L_{l, s, t}^{+}$and $L_{l, s, t}^{-}$are the adding and reducing amount of $l$ th shiftable load in the $s$ th scenario at time $t$ and $p_{l, t}^{+}$and $p_{l, t}^{-}$ are the price of shiftable load for adding and reducing load, respectively.

The day-ahead scheduling is subjected to the following constraint.

Power balance constraint:

$$
\sum_{i=1}^{N_{G}} P_{i, s, t}+W_{s, t}-W C_{s, t}=D_{s, t}+\sum_{l=1}^{N_{L}}\left(L_{l, s, t}^{+}-L_{l, s, t}^{-}\right)
$$

where $W_{s, t}, W C_{s, t}$, and $D_{s, t}$ is the wind power, wind curtailment, and load demand in the sth scenario at time $t$, respectively.

Power limit constraint of thermal generators:

$$
u_{i, t} \cdot P_{i}^{\min } \leq P_{i, s, t} \leq u_{i, t} \cdot P_{i}^{\max }
$$

where $u_{i, t}$ is the on/off state of $i$ th generator at time $t$. On/off state constraint [10]:

$$
\left\{\begin{array}{l}
x_{i, t}-y_{i, t}=u_{i, t}-u_{i, t-1} \\
x_{i, t}+y_{i, t} \leq 1
\end{array}\right.
$$

where $x_{i, t}$ and $y_{i, t}$ are, respectively, the start-up and shut-down variable of $i$ th generator at time $t$.

Ramping limit constraint:

$$
-R D_{i} \leq P_{i, s, t}-P_{i, s, t-1} \leq R U_{i},
$$

where $R D_{i}$ and $R U_{i}$ are ramp-down and ramp-up rate limit of $i$ th generator.

Power limit constraint of shiftable load:

$$
\begin{aligned}
& 0 \leq L_{l, s, t}^{+} \leq L_{l, s, t}^{\max +}, \\
& 0 \leq L_{l, s, t}^{-} \leq L_{l, s, t}^{\max -} .
\end{aligned}
$$

Energy constraint of shiftable load:

$$
\begin{aligned}
& E_{l, s, t}=\sum_{\tau=1}^{t}\left(L_{l, s, \tau}^{+}-L_{l, s, \tau}^{-}\right), \\
& E_{l, s, 1}=E_{l, s, N_{T}} .
\end{aligned}
$$

Constraints (9) and (10) indicate the curtailed load is shifted to other periods without energy loss.

2.3. Intraday Scheduling. The intraday scheduling adopts chance constraint programming. The intraday scheduling aims at minimizing the operation cost. The objective function can be formulized as follows:

$$
\min \left(f_{\mathrm{ID}}\right)=f_{\mathrm{G}}+f_{\mathrm{SL}}+f_{R}
$$

where the generation cost $f_{\mathrm{G}}$ can be written as follows:

$$
f_{G}=\sum_{i=1}^{N_{G}} \sum_{t=1}^{N_{T}}\left(a_{i}+b_{i} P_{i, t}+c_{i} P_{i, t}^{2}\right) \text {. }
$$

The shiftable load cost $f_{\mathrm{SL}}$ can be defined as follows:

$$
f_{\mathrm{SL}}=\sum_{l=1}^{N_{L}} \sum_{t=1}^{N_{T}}\left(p_{l, t}^{+} \cdot L_{l, t}^{+}+p_{l, t}^{-} \cdot L_{l, t}^{-}\right) \text {. }
$$

The reserve cost $f_{\mathrm{R}}$ can be defined as follows:

$$
f_{R}=\sum_{i=1}^{N_{G}} \sum_{t=1}^{N_{T}}\left(q_{l, t} \cdot K_{l, t}\right)
$$

The intraday scheduling is subjected to the following constraint.

Power balance constraint:

$$
\sum_{i=1}^{N_{G}} P_{i, t}+W_{t}-W C_{t}=D_{t}+\sum_{l=1}^{N_{L}}\left(L_{l, t}^{+}-L_{l, t}^{-}\right) .
$$

Power limit constraint of thermal generators:

$$
u_{i, t} \cdot P_{i}^{\min } \leq P_{i, t} \leq u_{i, t} \cdot P_{i}^{\max }
$$

Ramping constraint of thermal generators:

$$
-R D_{i} \leq P_{i, t}-P_{i, t-1} \leq R U_{i}
$$

Reserve constraint:

$$
\begin{array}{r}
\sum_{i=1}^{N_{G}} R_{i, t} \geq \Phi^{-1}(\eta) \sqrt{\delta_{D}^{2}+\delta_{W}^{2}}, \\
R_{i, t} \leq P_{i}^{\max }-P_{i, t},
\end{array}
$$

where $R_{i, t}$ is the reserve provided by the $i$ th generator at time $t, \eta$ is a confidence value, $\Phi^{-1}$ is the inverse distribution function of load and wind power, and $\delta_{D}$ and $\delta_{W}$ are the standard deviation of load forecasting and wind power forecasting, respectively.

Power limit constraint of shiftable load: 


$$
\begin{aligned}
& 0 \leq L_{l, t}^{+} \leq L_{l, t}^{\max +} \\
& 0 \leq L_{l, t}^{-} \leq L_{l, t}^{\max -}
\end{aligned}
$$

Note that the proposed method considers the wholehorizon period of the scheduled day, whereas the traditional method only considers the next 2 hours. So the difference between the proposed method and the traditional method is the energy constraint of the shiftable load.

The energy constraint of shiftable load for the proposed method is defined as follows:

$$
\begin{aligned}
E_{l, t} & =E_{l, t 0}+\sum_{\tau=1}^{t}\left(L_{l, \tau}^{+}-L_{l, \tau}^{-}\right), \\
E_{l, t 0 s} & =E_{l, N_{T}},
\end{aligned}
$$

where $E_{l, t 0}$ is the energy of the shiftable load at the beginning of the scheduling period and $E_{l, t 0 \mathrm{~s}}$ is the initial energy at the beginning of the day.

The difference between the proposed rolling scheduling method and traditional method lies in the intraday scheduling period $t \in\left[1, N_{\mathrm{T}}\right]$. In the traditional methods, the intraday scheduling period $N_{\mathrm{T}}$ is a fixed value, whereas the proposed method defines $N_{\mathrm{T}}$ as the time periods from the current time to the end of the day. By this way, the intraday scheduling can take into account the cost of the whole scheduling horizon.

\section{Testing Results}

The testing examples consider a power system with 5 individual generators and 2 load aggregators. Each load aggregator participates in the load shifting with different prices. The parameters of the generators and the shiftable load are listed in Tables 1 and 2, respectively. The forecasted curve of load and wind power is shown in Figure 2.

To perform scenario-based day-ahead scheduling, the wind scenarios are generated. The Monte Carlo method is firstly adopted to generate hundreds of scenarios, and then, the K-means clustering method is used to decrease the number of scenarios in order to the final 3 scenarios, in order to reduce the computational burden. The final wind scenarios are shown in Figure 3(a), based on which the scheduling results of different resources are obtained. The average power of scheduled power of different resources is shown in Figure 3(b).

The intraday scheduling utilizes more accurate intraday wind forecasting results, and more accurate scheduling results can be obtained. The intraday forecasted wind power is shown in Figure 4(a), and the intraday scheduling results of the next 2 hours are shown in Figures 4(b) and 4(c), where Figure 4(b) considers the whole-horizon intraday scheduling and Figure 4(c) only considers next 2-hour intraday
Table 1: Parameters of the generators.

\begin{tabular}{lccccc}
\hline & $\begin{array}{c}a \\
(\$)\end{array}$ & $\begin{array}{c}b \\
(\$ / \mathrm{MW})\end{array}$ & $\begin{array}{c}c \\
\left(\$ / \mathrm{MW}^{2}\right)\end{array}$ & $\begin{array}{c}P_{\max } \\
(\mathrm{MW})\end{array}$ & $\begin{array}{c}P_{\min } \\
(\mathrm{MW})\end{array}$ \\
\hline Generator 1 & 100 & 14 & 0.01 & 110 & 0 \\
Generator 2 & 100 & 15 & 0.01 & 100 & 0 \\
Generator 3 & 100 & 30 & 0.01 & 520 & 200 \\
Generator 4 & 100 & 35 & 0.01 & 200 & 0 \\
Generator 5 & 100 & 10 & 0.01 & 600 & 200 \\
\hline
\end{tabular}

scheduling. It can be seen that the whole-horizon intraday scheduling model can utilize shiftable load, whereas the next 2-hour intraday scheduling cannot. It is because the horizon intraday scheduling can reflect the whole-day benefit, whereas the next 2-hour intraday scheduling only reflects the benefit of net 2 hours.

Then, the performance of the proposed rolling scheduling method is examined. For comparison, 3 models are tested.

(1) Method 1: nonrolling scheduling method. All the resources are allocated in the day-ahead scheduling.

(2) Method 2: traditional rolling scheduling method. The scheduling period of the intraday scheduling is fixed and set to $2 \mathrm{~h}$.

(3) Method 3: the proposed rolling scheduling method. The scheduling period of the intraday scheduling is from the current time to the end of the day.

The scheduling results of different resources by Methods 1-3 are shown in Figure 5, in which the scheduling results of shiftable load are shown in Figure 6. From Figures 5 and 6, it can be seen that different resources are optimized by the scheduling methods. However, traditional rolling scheduling method (Method 2) utilizes very little scheduled shiftable load, whereas the proposed method (Method 3) and the nonrolling method (Method 1) utilize a similar amount of shiftable load.

To further show contribution of thermal units and shiftable load in the intraday scheduling, Figure 7(a) illustrates the difference between day-ahead and in-day forecasting error. Figures 7(b) and 7(c) show the adjusted power of thermal units and shiftable load by Method 2 and Method 3. It can be seen that the traditional rolling scheduling method (Method 2) adjusts the scheduled power much more than the proposed method (Method 3) in the intraday scheduling and is more difficult to be realized.

Table 3 lists the total operational cost of the 3 methods. It can be seen that the nonrolling scheduling method (Method 1) results in highest cost, since Method 1 has a higher forecasting error and higher cost of reserve. The traditional rolling scheduling method (Method 2) has a lower total operational cost. And the proposed method (Method 3) results in lowest operational cost among the 3 methods, exhibiting advantageous over the other 2 methods. 
TABle 2: Parameters of the shiftable load.

\begin{tabular}{llcccc}
\hline & & Price for upregulation (\$) & Price for downregulation (\$) & $\begin{array}{c}\text { Capacity for } \\
\text { upregulation }\end{array}$ & $\begin{array}{c}\text { Capacity for } \\
\text { downregulation }\end{array}$ \\
\hline \multirow{2}{*}{ Aggregator 1 } & Block 1 & 0 & 5 & $2.4 \%$ of the total load & $2.4 \%$ of the total load \\
& Block 2 & 0 & 8 & $1.2 \%$ of the total load & $1.2 \%$ of the total load \\
\hline \multirow{2}{*}{ Aggregator 2 } & Block 1 & 0 & 4 & $2.4 \%$ of the total load & $2.4 \%$ of the total load \\
& Block 2 & 0 & 7 & $1.2 \%$ of the total load & $1.2 \%$ of the total load \\
\hline
\end{tabular}

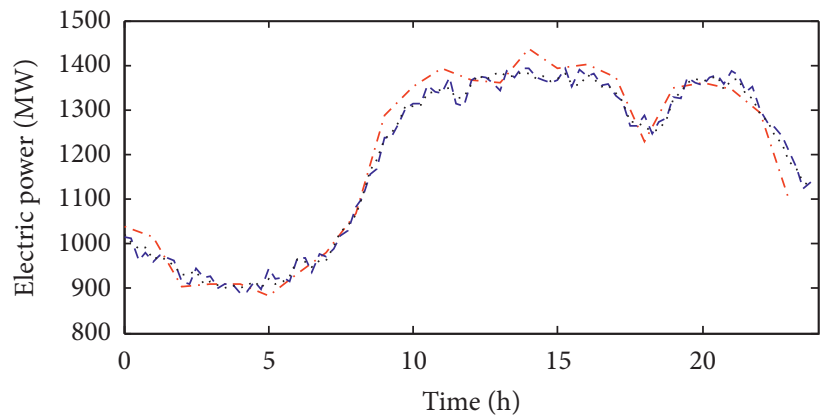

-.. Day-ahead forecasting

- - - In-day forecasting

..... Real curve

(a)

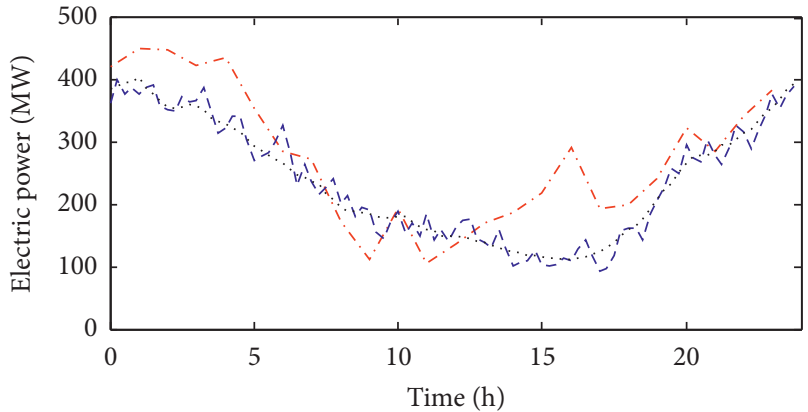

-.. Day-ahead forecasting

- - - In-day forecasting

..... Real curve

FIgURE 2: Forecasted curve of load and wind power: (a) forecasted load; (b) forecasted wind power.

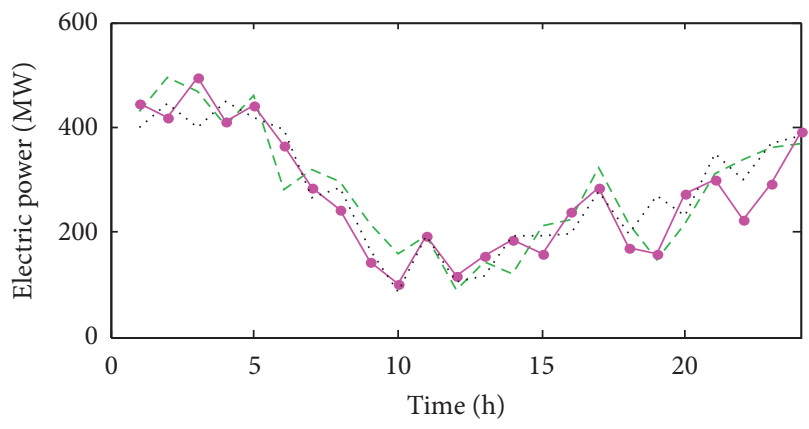

(a)

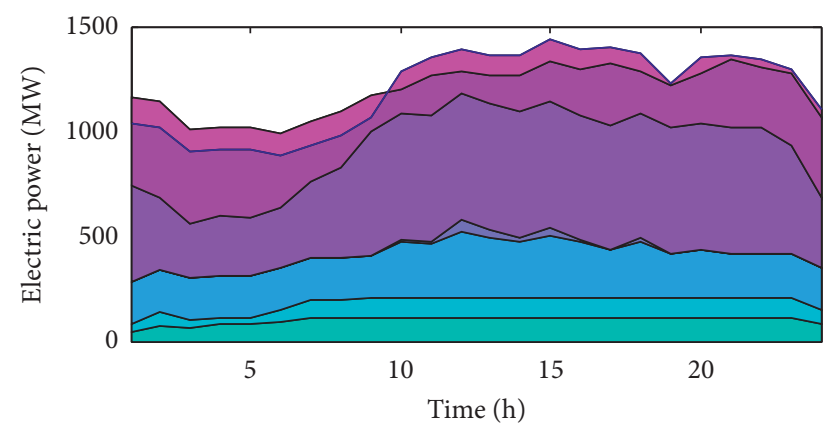

(b)

FIGURE 3: Day-ahead scheduling results: (a) wind scenarios; (b) average scheduled power of different resources.
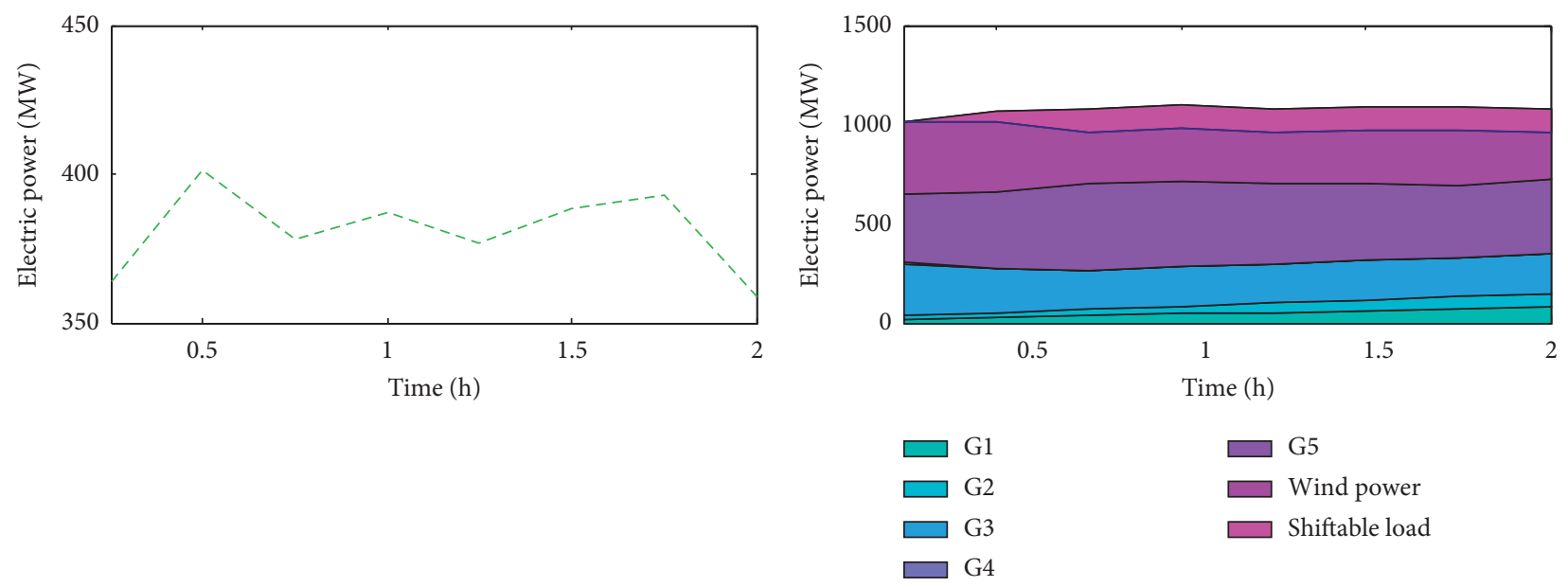

(a)

(b)

Figure 4: Continued. 


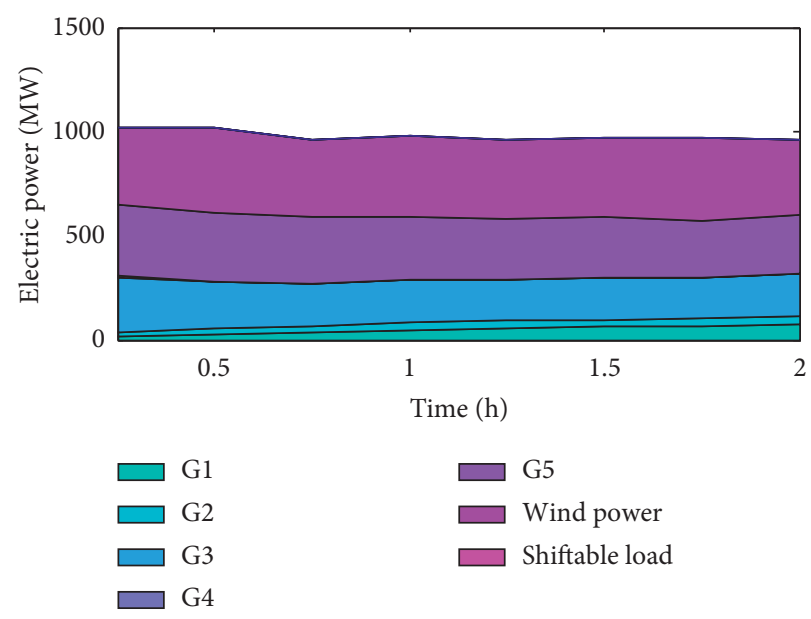

(c)

FIGURE 4: Intraday scheduling results of the next 2 hours: (a) forecasted wind power; (b) whole-horizon intraday scheduling; (c) next 2-hour intraday scheduling without considering the whole horizon.

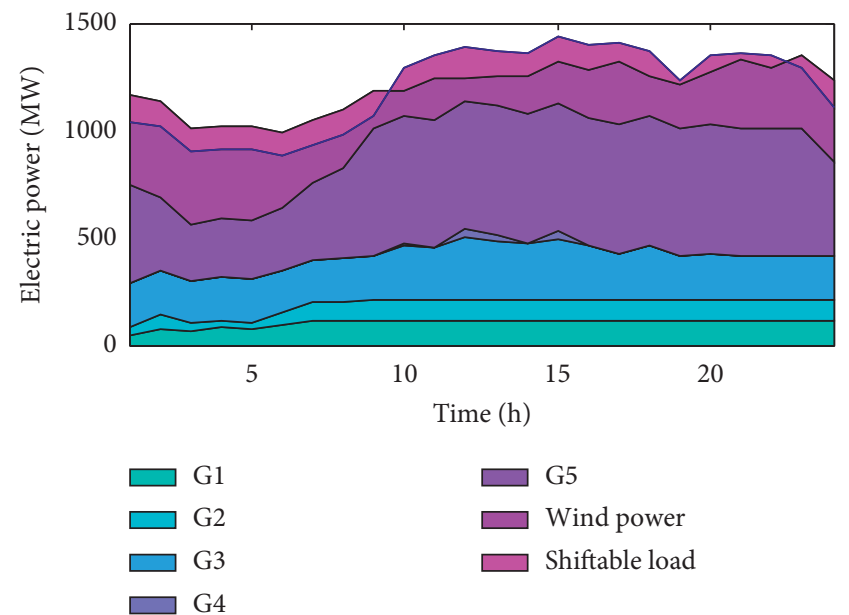

(a)

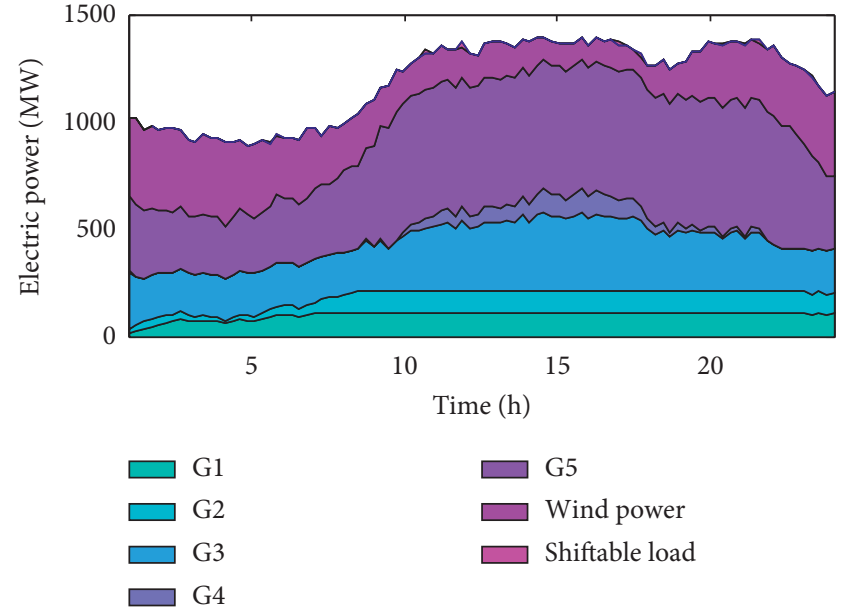

(b)

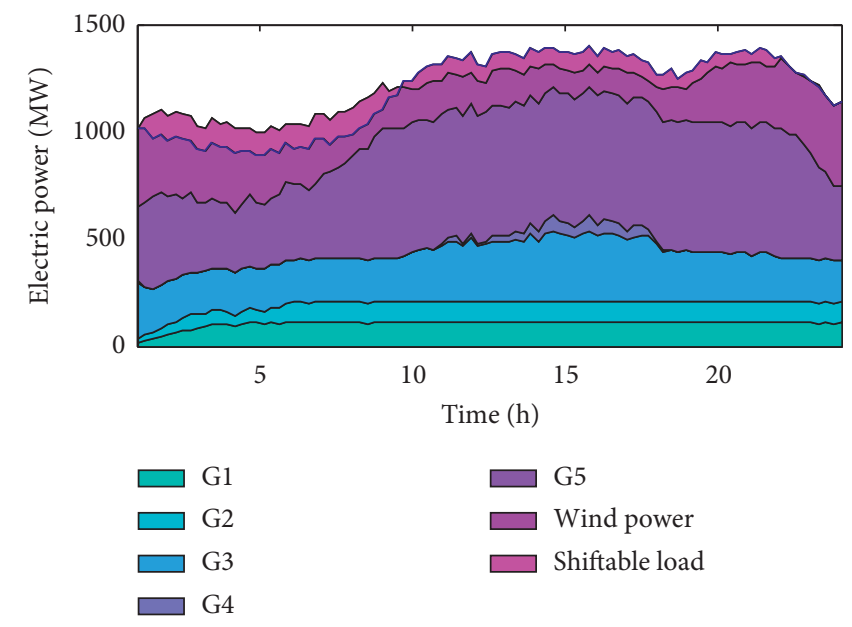

(c)

Figure 5: Scheduling results of different resources. (a) Method 1. (b) Method 2. (c) Method 3 (the proposed method). 


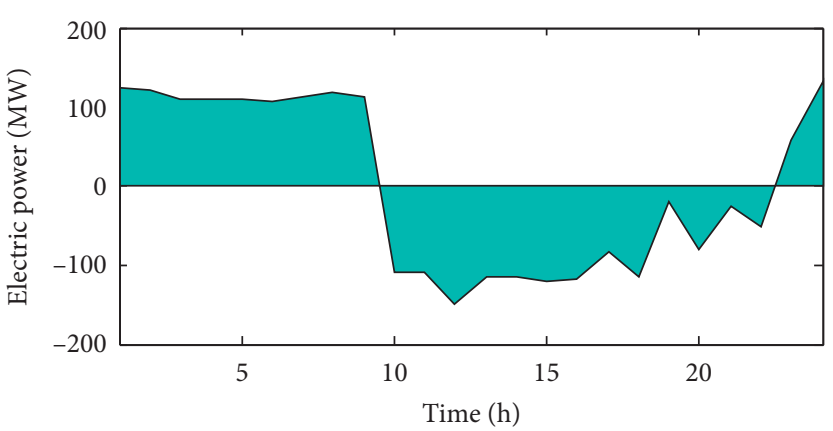

(a)

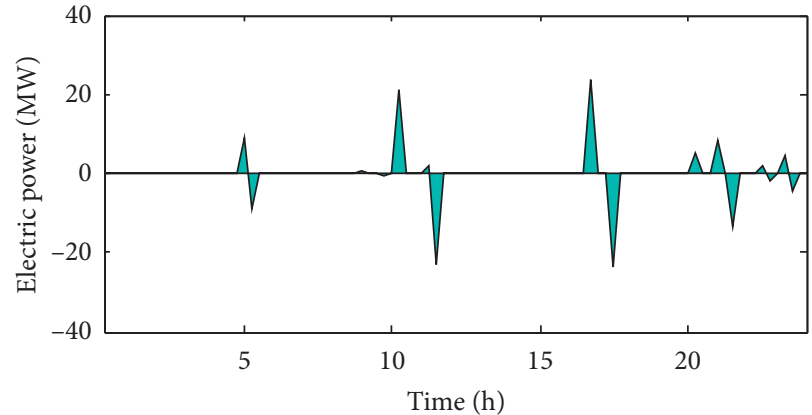

(b)

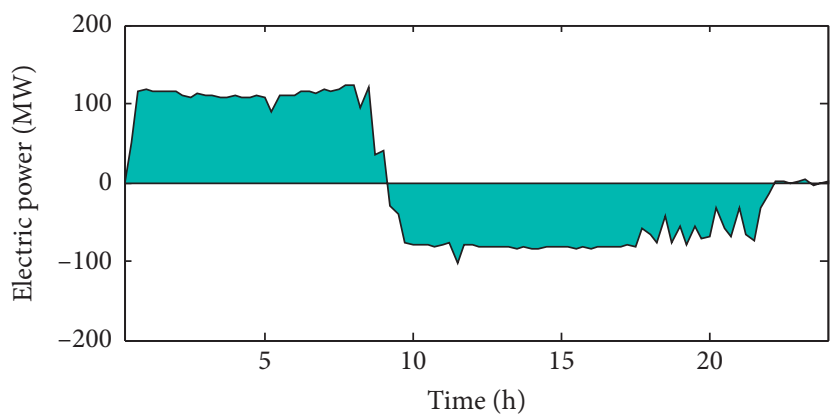

(c)

FIgURE 6: Scheduling results of shiftable load. (a) Method 1. (b) Method 2. (c) Method 3 (the proposed method).

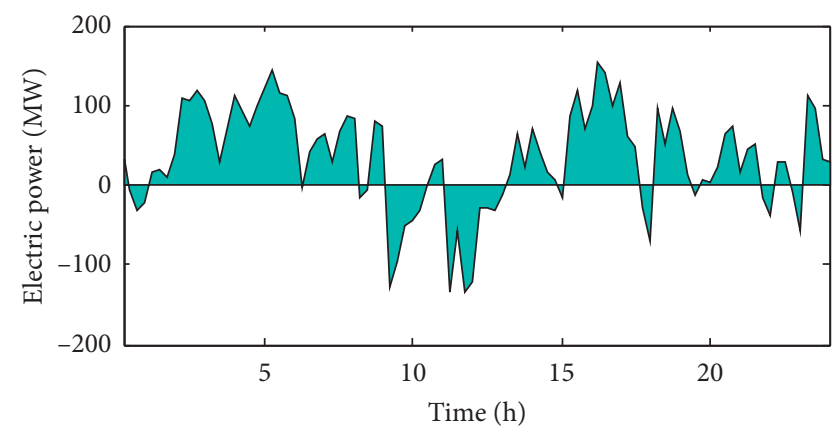

(a)

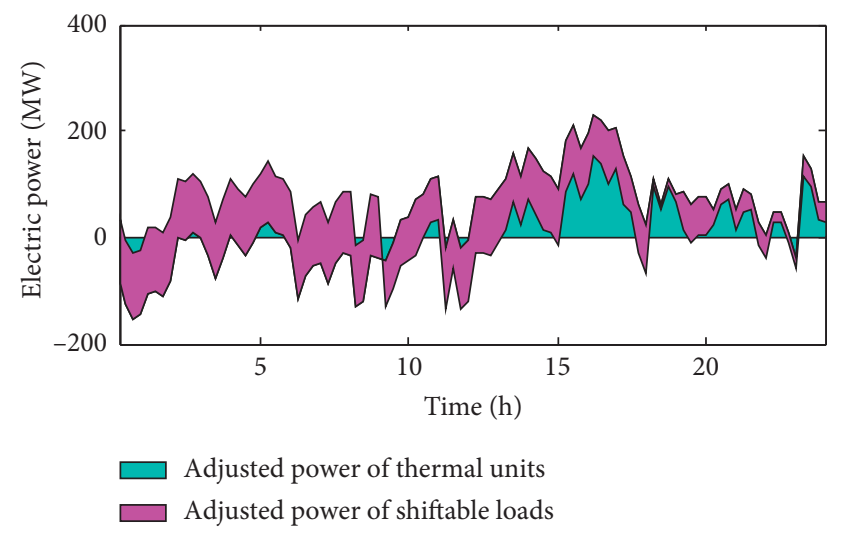

(b)

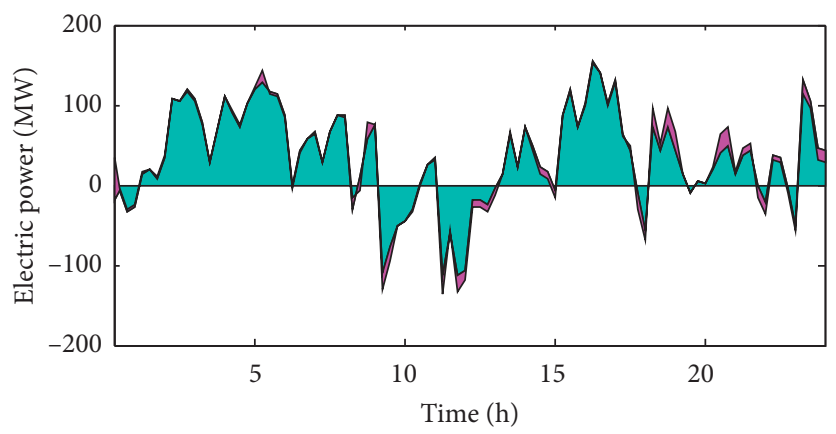

Adjusted power of thermal units

Adjusted power of shiftable loads

(c)

Figure 7: Adjusted power in the intraday scheduling. (a) Difference between day-ahead and intraday forecasting error. (b) Adjusted power of Method 2. (c) Adjusted power of Method 3 (the proposed method). 
TABLE 3: The total operational cost of different scheduling models.

\begin{tabular}{lc}
\hline & Operational cost $(\$)$ \\
\hline Method 1 & 491346 \\
Method 2 & 489106 \\
Method 3 & 475398 \\
\hline
\end{tabular}

\section{Conclusion}

This paper proposes an improved rolling scheduling framework that considers shiftable loads with intraday energy storage constraints. The key findings of this paper can be summarized as follows:

(1) By adopting the day-ahead intraday rolling scheduling strategy, the shiftable load and generating resources are successfully scheduled.

(2) By using the remaining time of the day as the intraday scheduling period, the scheduling can reflect the long-term benefits and therefore reduce the overall operation costs.

Future work may be applying other flexible resources, such as electrical vehicles and battery storages, in the proposed rolling scheduling model. Similar to shiftable loads, electrical vehicles and battery storages have the energy constraints, but the charging/discharging characteristic and the lifetime cost are quite different. Therefore, the future work may focus on modelling these characteristics and develop a proper rolling scheduling approach for them.

\section{Data Availability}

All data generated or analyzed during this study are included in this article.

\section{Conflicts of Interest}

The authors declare that they have no conflicts of interest.

\section{Acknowledgments}

This study was supported by the Science and Technology Project of China Southern Power Grid Corporation (090000KK52190230).

\section{References}

[1] X. Xu, Z. Y. An, M. Shahidehpour et al., "Data-driven riskaverse two-stage optimal stochastic scheduling of energy and reserve with correlated wind power," IEEE Transactions on Sustainable Energy, vol. 11, no. 1, pp. 436-447, 2020.

[2] V. Trovato, F. Teng, and G. Strbac, "Role and benefits of flexible thermostatically controlled loads in future low-carbon systems," IEEE Transactions on Smart Grid, vol. 9, no. 5, pp. 5067-5079, 2018.

[3] A. Radaideh, U. Vaidya, and V. Ajjarapu, "Sequential setpoint control for heterogeneous thermostatically controlled loads through an extended Markov chain abstraction," IEEE Transactions on Smart Grid, vol. 10, no. 1, pp. 116-127, 2017.

[4] H. Qiu, W. Gu, Y. Xu et al., "Multi-time-scale rolling optimal dispatch for AC/DC hybrid microgrids with day-ahead distributionally robust scheduling," IEEE Transactions on Sustainable Energy, vol. 10, no. 4, pp. 1653-1663, 2019.

[5] H. Yang, M. Li, Z. Jiang et al., "Multi-time scale optimal scheduling of regional integrated energy systems considering integrated demand response," IEEE Access, vol. 8, pp. 50805090, 2020.

[6] Q. Yuan, Y. Wu, B. Li et al., "Multi-timescale coordinated dispatch model and approach considering generation and load uncertainty," Power System Protection and Control, vol. 47, no. 16, pp. 8-16, 2019.

[7] Y. Song, Y. Liu, R. Wang et al., "Multi-objective configuration optimization for isolated microgrid with shiftable loads and mobile energy storage," IEEE Access, vol. 7, pp. 95248-95263, 2019.

[8] L. Zhu, X. Zhou, X. Zhang et al., "Integrated resources planning in microgrids considering interruptible loads and shiftable loads," Journal of Modern Power Systems and Clean Energy, vol. 6, no. 4, pp. 802-815, 2018.

[9] I. Avramidis, F. Capitanescu, and G. Deconinck, "Practical approximations and heuristic approaches for managing shiftable loads in the multi-period optimal power flow framework," Electric Power Systems Research, vol. 190, Article ID 106864, 2021.

[10] Y. Dvorkin, H. Pandžić, M. A. Ortega-Vazquez, and D. S. Kirschen, "A hybrid stochastic/interval approach to transmission-constrained unit commitment," IEEE Transactions on Power Systems, vol. 30, no. 2, pp. 621-631, 2015.

[11] C. Zhao, J. Wang, J. Watson, and Y. Guan, "Multi-stage robust unit commitment considering wind and demand response uncertainties," IEEE Transactions on Power Systems, vol. 28, no. 3, pp. 2708-2717, 2013. 